\title{
Methacarn Fixation: A Novel Tool for Analysis of Gene Expressions in Paraffin-Embedded Tissue Specimens
}

\author{
Makoto Shibutani, Chikako Uneyama, Keiko Miyazaki, Kazuhiro Toyoda, and \\ Masao Hirose \\ Division of Pathology, National Institute of Health Sciences, Kamiyoga, Setagaya-ku, Tokyo, Japan
}

\begin{abstract}
SUMMARY: To establish a quantitative method for analysis of gene expressions in small areas of tissue after paraffin embedding, preliminary validation experiments with RT-PCR and Western blotting were performed using methacarn-fixed rodent tissues and a cultured PC12 cell line. A total RNA yield of $52 \pm 15 \mathrm{ng} / \mathrm{mm}^{2}$, sufficient for a quantitative RT-PCR of many genes, could be extracted from a deparaffinized $10-\mu \mathrm{m}$-thick rat-liver section by a simple, single-step extraction method. The low concentration of contaminating genomic DNA and the resolution of ribosomal RNAs in RNA gel proved the purity and integrity of the extracted RNA samples, allowing PCR amplification of a long mRNA sequence and mRNA species expressing low copy numbers. PCR amplification of mRNA-derived target gene fragments could be achieved by optimizing the amount of total RNA for reverse transcription and the number of subsequent PCR cycles for each gene. By this validation, organ- and sex-specific mRNA expression could be detected in methacarn-fixed paraffin-embedded tissues without additional DNase treatment of RNA samples. RT-PCR analysis could also be performed with total RNA extracted from deparaffinized tissue dissected with a laser capture microdissection system. In addition, extraction of protein yielded $4.9 \pm 2.1 \mu \mathrm{g} / \mathrm{mm}^{2}$ from a $10-\mu$ m-thick rat-liver section, allowing a quantitative expression analysis of protein by Western blotting. Thus, in addition to its advantages for immunohistochemistry, methacarn-fixed paraffin-embedded tissue has benefits for analysis of both RNAs and proteins in the cells of histologically defined areas. (Lab Invest 2000, 80:199-208).
\end{abstract}

Q uantitative analysis of gene expression in pathologically altered cell populations can provide valuable information regarding the mechanisms underlying biological phenomena, such as inflammatory responses, cell growth, differentiation, and apoptotic cell death. For pathological assessment, paraffin embedding is routinely used because of the ease of handling tissues and subsequent staining, as well as good preservation of morphology. If paraffin-embedded tissue (PET) retains RNAs and proteins intact, then it can be applied as a material source for defined cell populations, for example by microdissection.

Until now, extraction of total RNA and its analysis have been extensively performed using formalin-fixed PET for the detection of RNA viruses such as hepatitis C (Dries et al, 1999; Guerrero et al, 1997, 1998; Miyauchi et al, 1998). However, degradation of RNA species or insufficient extraction efficiency due to incomplete lysis are problems (Finke et al, 1993; Rupp and Locker, 1988; Stanta and Schneider, 1991), and, therefore, quantitative analysis of specific gene expression cannot be applied. Several alternative fixatives have been tested for their performance with RT-PCR analysis, but there has not been sufficient validation for practical RT-PCR experiments because

Received October 25, 1999.

This work was supported by a grant-in-aid from the Ministry of Health and Welfare of Japan (Grant H11-Seikatsu-74).

Address reprint requests to: Dr. M. Shibutani, Division of Pathology, National Institute of Health Sciences, 1-18-1, Kamiyoga, Setagaya-ku, Tokyo 158-8501. Fax: 8133700 1425; E-mail: shibutan@nibs.go.jp of contaminating genomic DNA. To avoid the noise from such contamination, either DNase-treatment of extracted total RNA or primer design allowing for the distinction of PCR products derived from genomic DNA and mRNA are necessary (Foss et al, 1994). However, DNase-treatment with subsequent phenol/ chloroform extraction and ethanol precipitation of extracted RNA samples may reduce final RNA yield considerably, and genomic DNA information is not always available. Therefore, establishment of a simple method for extraction and analysis of RNA that can overcome the problem of contaminating DNA is of high priority.

Compared with RNA analysis, little is known about the potential for protein extraction from histopathological material in PET (Hara et al, 1993; Ikeda et al, 1998), because formaldehyde crosslinks proteins. AMeX fixation does not affect the quality of protein, but this method is laborious and requires special equipment without offering any advantages in terms of RNA preservation (Sato et al, 1991, 1992).

If an antigen is properly immobilized, non-crosslinking protein-precipitating fixatives usually give superior immunohistochemical results than aldehydebased cross-linking fixatives (Banks, 1979; Orstavik et al, 1981; Rognum et al, 1980), because antigenicity is usually maintained intact (Mitchell et al, 1985). We found that, after fixation with the protein-precipitating methacarn fixative, deparaffinized tissues were easily solubilized by protein detergents or by a phenol solution. The individual constituents of methacarn did not seem to affect the primary structure of polynucleoti- 
des, so that RNA molecules as well as proteins could be extracted from methacarn-fixed PET.

In this study, we applied a simple extraction method for total RNA and protein to methacarn-fixed PET and found that mRNA-specific PCR amplification and immunoblot detection of proteins appropriate for quantitative analysis could be performed with small tissue specimens.

\section{Results}

\section{Yield and Quality of Total RNA}

The lower limit of RNA titration with RiboGreen fluorescent dye was $5 \mathrm{ng} / \mathrm{ml}$. Yields of total RNA extracted from unfixed frozen PC12 cells and methacarn-fixed paraffin-embedded PC12 cells were $9.24 \pm 0.17$ and $5.13 \pm 1.30 \mu \mathrm{g} / 5 \times 10^{5}$ cells, respectively $(n=5)$. RNA yield from the paraffin-embedded cells, however, was underestimated because cell pellets could not be formed by a Microfuge in xylene, and many cells were lost during deparaffinization. Total RNA could not be extracted from deparaffinized paraformaldehyde-fixed cells. Similar results were also observed in the yield of total RNA from rat liver PET blocks when different fixatives were compared (Table 1). RNA yield from methacarn-fixed tissue was $0.74 \pm 0.11 \mu \mathrm{g} / \mathrm{mg}$ wet weight, about $46 \%$ of that from unfixed frozen tissue. Contamination of genomic DNA in the RNA preparation from methacarn-fixed tissue was better than that from unfixed tissue. Although acetone showed the best RNA yield among fixatives, contamination of DNA was also high in the RNA preparation. Other fixatives, all containing formaldehyde as a constituent, did not give sufficient RNA yields. When the RNA yield per unit area of tissue section was measured, methacarn-fixed rat liver showed a yield of $52 \pm 15 \mathrm{ng} / \mathrm{mm}^{2}$ from a small tissue specimen (10 $\mu \mathrm{m}$ thick, $3.0 \mathrm{~mm}^{2}$ sized area, $n=$ 6). As shown in Figure 1, the integrity of total RNA extracted from methacarn-fixed paraffin-embedded PC12 cells was well preserved and similar to that from unfixed frozen cells, judging from the resolution of $18 \mathrm{~S}$ and $28 \mathrm{~S}$ ribosomal RNAs.

\section{Validation of RT-PCR}

Figure 2 shows results for RT-PCR validation using a primer set distinguishing PCR-products of genomic

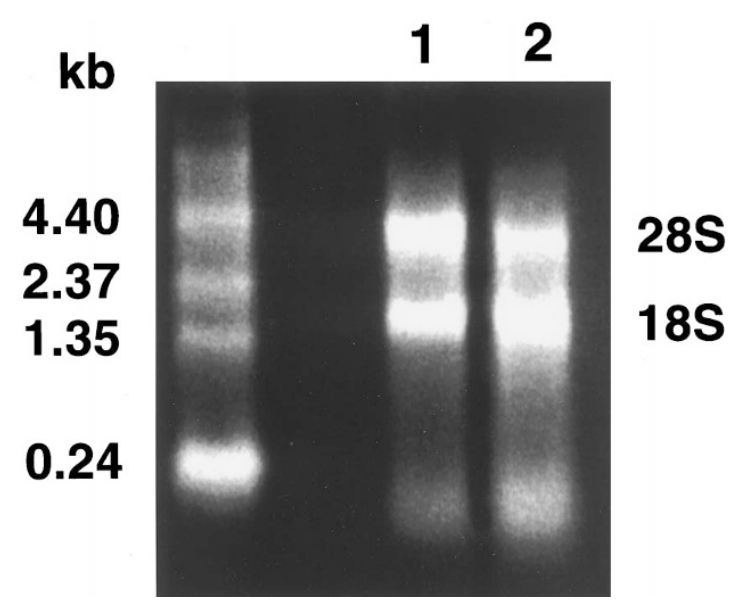

Figure 1.

Quality of total RNA extracted from methacarn-fixed, paraffin-embedded PC12 cells. Two- $\mu \mathrm{g}$ total RNA samples were resolved in a $0.8 \%$ formaldehydeagarose gel and visualized with ethidium bromide. Lane 1, unfixed frozen cells; Lane 2, methacarn-fixed paraffin-embedded cells.

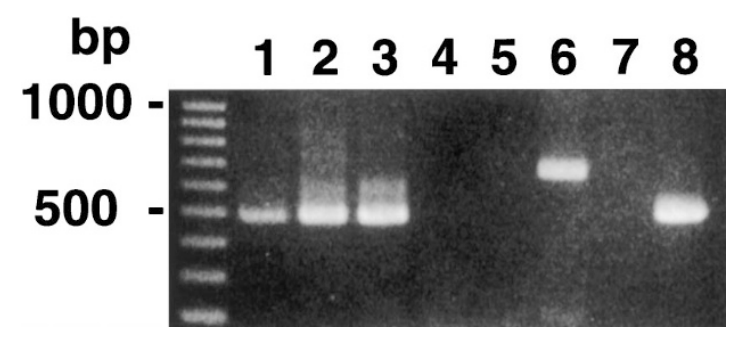

Figure 2.

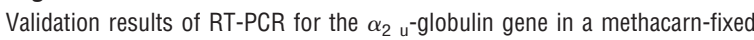
paraffin-embedded male rat liver section. The cycle number was set at 21 with $500 \mathrm{ng}$ of total RNA used for the RT reaction. Lane 1, methacarn-fixed liver section; Lane 2, methacarn-fixed liver section with DNase I treatment; Lane 3, unfixed frozen liver tissue; Lanes 4 and 5 , reverse transcriptase $(-)$ mock RT-PCR using $500 \mathrm{ng}$ (Lane 4) and one $\mu \mathrm{g}$ (Lane 5) of total RNA from methacarn-fixed liver sections; Lanes 6 and 7, positive and negative PCR controls using glyceraldehyde-3-phosphate dehydrogenase (GAPDH) primers with or without the GAPDH CDNA template, respectively; Lane 8, positive control of RT-PCR using in-vitro-transcribed mRNA of chloramphenicol acetyltransferase (CAT-RNA RT-PCR).

DNA and mRNA origins. In this figure, expression of $\alpha_{24}$-globulin RNA in male rat liver was validated using RT products obtained from 500-ng total RNA/20 $\mu \mathrm{l}$ total reaction. The optimum cycle number was prede-

Table 1. RNA-Yields and Concentrations of Contaminating DNA of the RNA Preparation Extracted from Rat Liver PETs

\begin{tabular}{lccc}
\hline \multicolumn{1}{c}{ Fixative } & No. of sample & $\begin{array}{c}\text { RNA yield } \\
(\mu \mathrm{g} / \mathrm{mg} \text { wet tissue })\end{array}$ & $\begin{array}{c}\text { DNA contamination } \\
(\mathrm{ng} / \mathrm{mg} \text { wet tissue })\end{array}$ \\
\hline Unfixed frozen & 6 & $1.55 \pm 0.38 \dagger$ & $5.85 \pm 1.31$ \\
Methacarn & 5 & $0.74 \pm 0.11^{*}$ & $0.78 \pm 0.20^{*}$ \\
Acetone & 5 & $0.82 \pm 0.13^{*}$ & $5.55 \pm 2.29 \dagger$ \\
Paraformaldehyde & 5 & $0.16 \pm 0.17^{*} \dagger$ & $1.12 \pm 0.55^{*}$ \\
Buffered formalin & 5 & $0.30 \pm 0.21^{*} \dagger$ & $0.75 \pm 0.51^{*}$ \\
Ufix & 5 & $0.05 \pm 0.10^{*} \dagger$ & $0.32 \pm 0.45^{*}$ \\
Bouin's solution & 5 & $0.14 \pm 0.14^{*} \dagger$ & $1.88 \pm 0.91^{*}$ \\
\hline
\end{tabular}

* Significantly different from unfixed frozen sample $(p<0.0001)$

$\dagger$ Significantly different from Methacarn-fixed sample $(p<0.005)$.

PET, paraffin-embedded tissue. 
termined to be 21 , under which condition the mRNAderived target fragment of $\alpha_{2 u}$-globulin could be amplified by the RT product from methacarn-fixed PET (lane 1) comparable to the unfixed frozen tissue case (lane 3). DNase I treatment of the extracted RNA sample did not affect the amplification (lane 2), and reverse transcriptase (-) mock RT did not amplify products (lanes 4 and 5).

For a gene whose genomic sequence is not available, a mock RT-PCR experiment with 35 cycles was performed to determine the maximum amount of total RNA for mRNA-specific gene amplification. Figure 3 shows validation results of PCR for hepatocyte growth factor receptor (HGFR) using mock RT products with $500 \mathrm{ng}$ and $1 \mu \mathrm{g}$ total RNA from the rat liver to estimate template amplification derived from contaminating genomic DNA. When 500 ng of total RNA was used, the mock RT-PCR product could not be detected, whereas it was present with one $\mu \mathrm{g}$ of total RNA. With $500 \mathrm{ng}$ of total RNA, the optimum cycle number for HGFR-RNA amplification was determined to be 27 .

Figure 4A shows RT-PCR results for several genes at 27-PCR cycles using RT products prepared from $500 \mathrm{ng}$ of liver total RNA from a male rat. RNA signals for HGFR and ribosomal protein L7 but not for the thyrotropin (TSH) $\beta$ subunit. TSH receptor (TSHR), prolactin (PRL), and growth hormone $(\mathrm{GH})$, could be detected as expected (Faliks and Meyuhas, 1982; Liu et al, 1996). Primer sets for TSH $\beta, \mathrm{PRL}$ and GH could amplify the respective mRNA-derived target fragments in rat pituitary total RNA, and TSHR primers in rat thyroid total RNA as well (data not shown). The origin of the PCR product from glyceraldehyde-3-

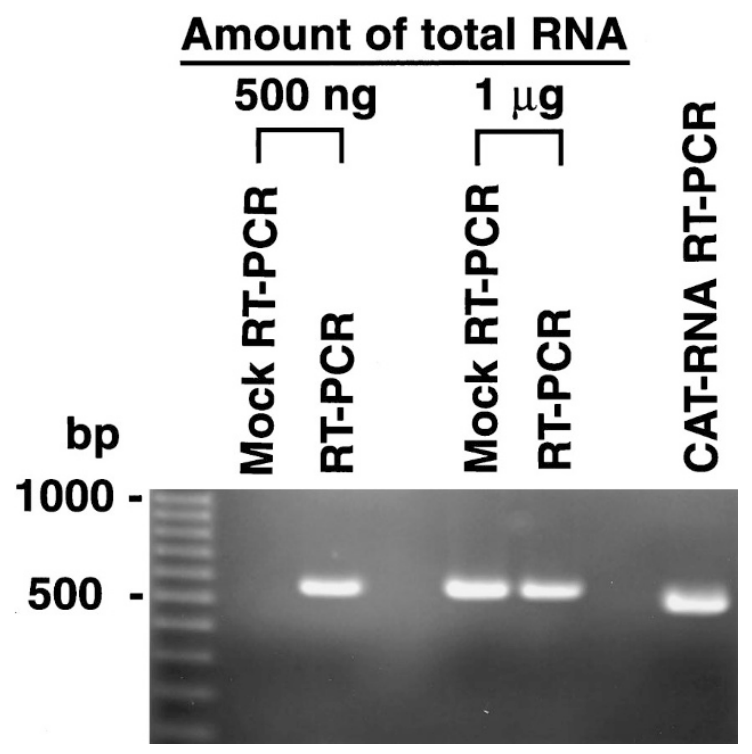

Figure 3.

Results of a validation mock RT-PCR experiment to estimate the maximum amount of total RNA for hepatocyte growth factor receptor (HGFR) RNAspecific amplification. PCR of 35 cycles was performed using reverse transcriptase (-) mock RT-product from $500 \mathrm{ng}$ and $1 \mu \mathrm{g}$ total RNA extracted from methacarn-fixed paraffin-embedded rat liver sections. Positive control for RT-PCR was performed as in Figure 2.
A

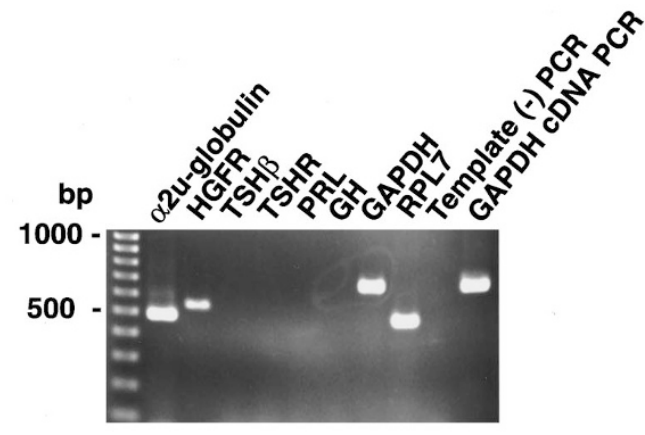

B

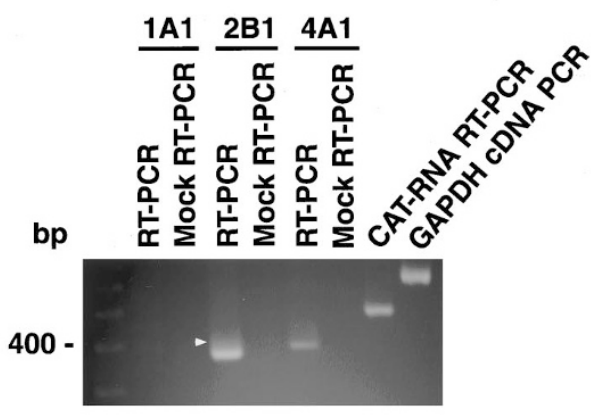

C

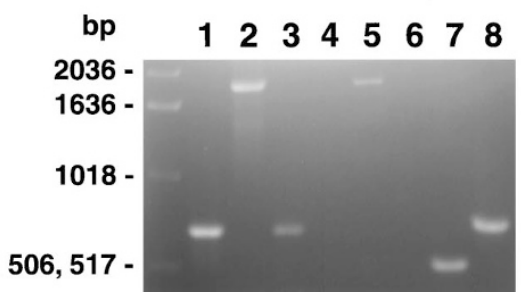

Figure 4.

RT-PCR results with methacarn-fixed paraffin-embedded rat-liver section. Panel A: The RT-reaction was performed using $500 \mathrm{ng}$ of total RNA from a male liver, followed by 27 cycles of PCR to detect RNA-expression of $\alpha_{2 \mathrm{u}}$-globulin, hepatocyte growth factor receptor (HGFR), thyrotropin subunit (TSH) $\beta$ subunit, TSH receptor (TSHR), prolactin (PRL), growth hormone (GH), GAPDH and ribosomal protein L7. Panel B: RT-PCR results of cytochrome P 450 (CYP)1A1, CYP2B1, CYP2B2 and CYP4A1. The RT reaction was performed using $500 \mathrm{ng}$ of total RNA, followed by 35 cycles of PCR. A primer set for CYP2B1 also amplifies a CYP2B2 RNA-derived CDNA template (arrowhead). Mock RT-PCR for each gene was performed as in Figure 3. Panel C: RT-PCR results of epidermal growth factor receptor (EGFR) RNA fragments of 693 base pairs; 1.9-kb PCR of 35 cycles was performed using RT product from $500 \mathrm{ng}$ total RNA with cycle parameters being $94^{\circ} \mathrm{C}$ for 1 minute, $58^{\circ} \mathrm{C}$ for 1 minute, and $72^{\circ} \mathrm{C}$ for 1 minutes 30 seconds. RT product was generated by a gene-specific primer (5'-AAGGCCTGGC CCAGCACATC-3'). Lanes 1 and 2, unfixed frozen liver tissue; Lanes 3-6, methacarn-fixed liver section; Lane 7, CAT-RNA RT-PCR; Lane 8, GAPDH CDNA PCR. Reverse transcriptase (-) mock RT-PCR was performed for each of the 693 base pairs (Lane 4) and the 1.9-kb (Lane 6) fragment.

phosphate dehydrogenase (GAPDH) PCR in this figure was obviously contaminating genomic DNA in this PCR condition, because it could be detected by mock RT-PCR at 27 cycles even with $125 \mathrm{ng}$ of total RNA from a liver section (data not shown), probably due to GAPDH-pseudogene products derived from contaminating genomic DNA (Hanauer and Mandel, 1984). Figure 4B shows RT-PCR results of cytochrome $\mathrm{P} 450$ (CYP), CYP2B1, CYP2B2 and CYP4A1, whose steady-state expression levels in the rat liver or hepa- 
tocytes are known to be extremely low unless xenobiotically stimulated (Kocarek et al, 1998; Omiecinski et al, 1990; Prough et al, 1994) In the present study, the steady-state expression levels of CYP2B1, CYP2B2 and CYP4A1 genes could be detected in the methacarn-fixed rat liver PET, while expression of the CYP1A1 gene was undetectable even at 35-PCR cycles. The pattern and intensity of these RNA signals from methacarn-fixed liver was equivalent to those in unfixed tissue (data not shown). In addition to mRNA species expressing low copy numbers, PCR amplification of an extended length of an mRNA sequence could also be achieved in methacarn-fixed PETs. Figure 4C shows a successful RT-PCR result of a 1.9-kilobase $(\mathrm{kb})$ fragment for epidermal growth factor receptor (EGFR) in the methacarn-fixed rat liver PET, despite the fact that the signal of the amplified product was weak compared with that from unfixed frozen tissue.

According to the prescribed PCR conditions, an accurate tissue distribution of specific gene expression could be analyzed. HGFR mRNA was also detected in the tissue sections from the brain, lungs and kidneys (Fig. 5A) as well as from the liver (Liu et al, 1996). On the other hand, $\alpha_{2 u}$-globulin mRNA was observed only in the liver (Fig. 5B) (lkeda et al, 1997).

\section{Protein Analysis}

Methacarn-fixed PET after deparaffinization could easily be solubilized in $2 \times$ sodium dodecyl sulfate

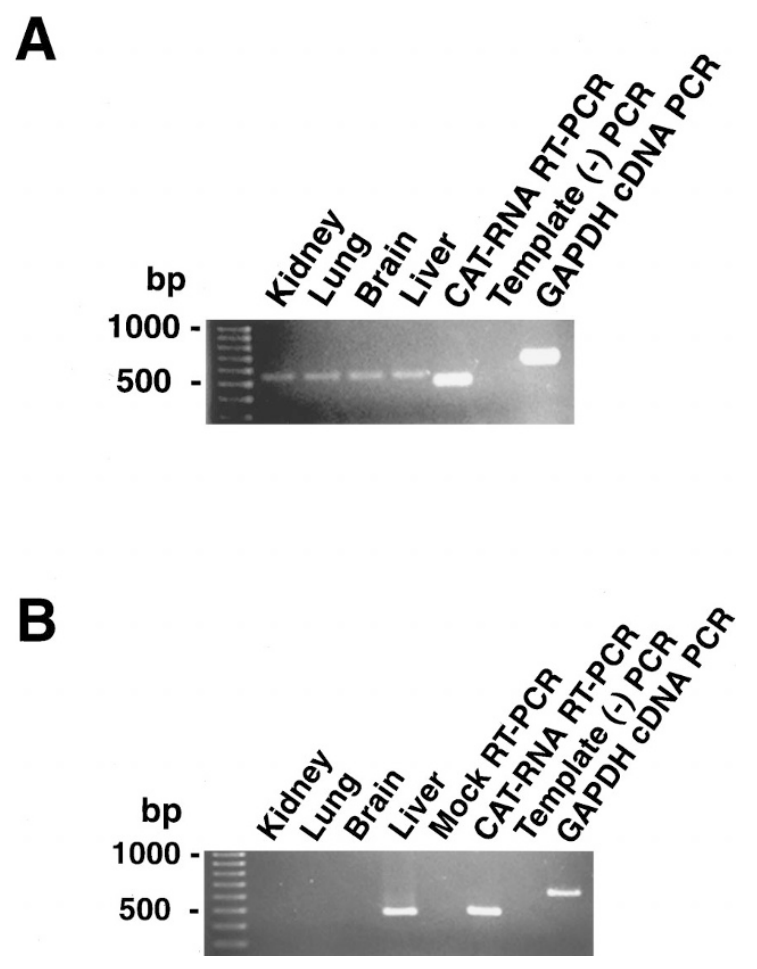

Figure 5.

Tissue distribution of HGFR and $\alpha_{2 u}$-globulin in male rat kidney, lung, brain and liver tissues. Panel A: HGFR; Panel B: $\alpha_{2 u}$-globulin. RT reactions were performed using $500 \mathrm{ng}$ total RNA from each tissue. PCR-cycle numbers for HGFR and $\alpha_{2 u}$-globulin were 27 and 21, respectively.
(SDS) gel-loading buffer by sonication. When protein yield from rat liver was compared, acetone-fixed PET block showed an equivalent yield to unfixed frozen tissue (Table 2). Methacarn-fixed PET resulted in $76.5 \%$ of the yield from unfixed tissue. In addition to total RNA yield, other fixatives containing formaldehyde as a constituent did not show sufficient protein yield. When the protein yield per unit area of tissue section was measured, methacarn-fixed rat liver showed a yield of $4.9 \pm 2.1 \mu \mathrm{g}$ protein extracts from a $1-\mathrm{mm}^{2}$ area of a $10-\mu \mathrm{m}$ section. Figure $6 \mathrm{~A}$ shows Western blotting data for $\alpha_{2 \mathrm{u}}$-globulin in the rat liver and kidney from both sexes. Alpha ${ }_{24}$-globulin was found specifically in the male liver (19 kDa) and kidney (17 kDa), whereas the mRNA was found only in the male liver (Fig. 6B). These results match well the following characteristic features of this protein: (a) Alpha $_{2 \mathrm{u}}$-globulin $(18.7 \mathrm{kDa})$ is synthesized mainly in the liver of the male rat (Alvares et al, 1996; Neuhaus, 1986, 1992); (b) Synthesized protein is secreted into the blood stream and rapidly excreted into urine (Neuhaus, 1986, 1992); (c) Excreted protein is reabsorbed into the proximal tubules of the kidney (Neuhaus, 1986, 1992); and (d) Absorbed $\alpha_{2 u}$-globulin is processed for proteolytic cleavage to become a 17kDa "kidney fatty acid-binding protein" (Ikeda et al, 1997).

As shown in Figure 7A, cathepsin D could also be detected in the ethinyl estradiol-treated mouse uterus (Moulton and Khan, 1992; Rochefort et al, 1989), and methacarn fixation and the following paraffin embedding did not affect extraction efficiency or integrity of the protein when compared with protein extracts isolated directly from the uterus. Signals of EGFR and CYP2E1 could also be detected in methacarn-fixed rat liver sections (Fig. 7, B and C). The EGFR signals from both methacarn-fixed and unfixed liver tissue migrated to the same position, size $170 \mathrm{kDa}$. The CYP2E1 polypeptide band migrated to the position of correct molecular size $(52 \mathrm{kDa})$ by precipitation of deparaffinized tissue with $10 \%$ trichloroacetic acid (TCA) before solubilization in $2 \times$ SDS gel-loading buffer. However, the shift in the molecular size of the signal (approximately $56 \mathrm{kDa}$ ) occurred when protein extracts were prepared without TCA precipitation. Relative band intensity of each protein from methacarn-fixed tissue samples per unfixed frozen

Table 2. Protein Yields from Rat Liver PETs

\begin{tabular}{lcc}
\hline \multicolumn{1}{c}{ Fixative } & No. of sample & $\begin{array}{c}\text { Yield of protein } \\
(\mu \mathrm{g} / \mathrm{mg} \text { wet tissue })\end{array}$ \\
\hline Unfixed frozen & 4 & $179 \pm 21$ \\
Methacarn & 5 & $137 \pm 26$ \\
Acetone & 5 & $175 \pm 41$ \\
Paraformaldehyde & 5 & $19 \pm 4^{*} \dagger$ \\
Buffered formalin & 5 & $23 \pm 5^{*} \dagger$ \\
Ufix & 5 & $21 \pm 5^{*} \dagger$ \\
Bouin's solution & 5 & $111 \pm 75$ \\
\hline
\end{tabular}

* Significantly different from unfixed frozen sample $(p<0.0001)$.

† Significantly different from Methacarn-fixed sample $(p<0.0001)$. 
A

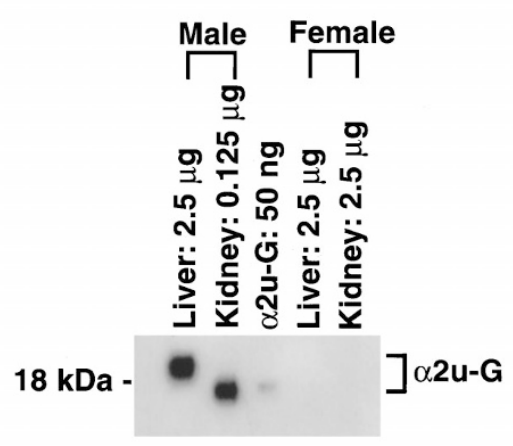

B

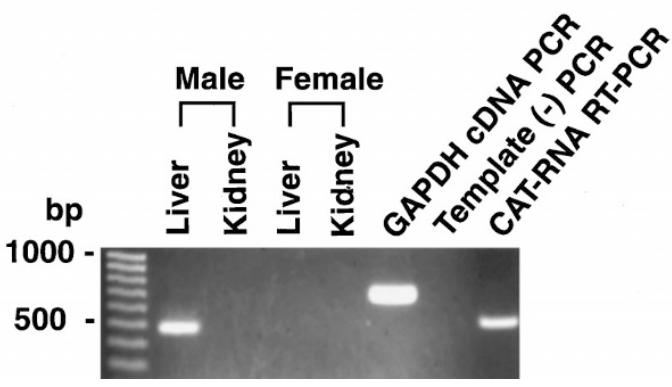

Figure 6 .

Comparison of protein and RNA expression in rat liver and kidney of both sexes. Both protein extracts and total RNA were obtained from methacarnfixed PETs. A, Male-specific expression of $\alpha_{2 u}$-globulin in the rat liver and kidney. B, RT-PCR detection of $\alpha_{2 u}$-globulin RNA in the same tissues as shown in Panel A. $\alpha_{2 u}$-G: purified $\alpha_{2 u}$-globulin from male rat urine.

samples were as follows: EGFR, $101.1 \pm 30.6 \%(n=$ 4); CYP2E1, $101.9 \pm 21.9 \%(n=5)$. The EGFR-signal could also be detected in acetone-fixed liver PET, and the intensity and integrity of this protein were equivalent to those in unfixed frozen tissue (data not shown).

\section{Laser Capture Microdissection RT-PCR}

As a preliminary study of laser capture microdissection (LCM) RT-PCR, rat liver tissue sections of different thickness were prepared and used for capture with different laser shot numbers (100-1000 shots), spot size $(30 \sim 60 \mu \mathrm{m})$ and amplitude (30-100 watts [w]). Although the RNA yields fluctuated considerably, thick sections between 8 and $10 \mu \mathrm{m}$ could yield $5-70 \mathrm{ng}$ of total RNA with 100-1000 shots of $60 \mu \mathrm{m}$ spot size and 60-w amplitude. Figure 8 shows successful LCM-RTPCR results for HGFR from 47 and $15 \mathrm{ng}$ total RNA extracted from cresyl violet-stained liver tissue dissected by the LCM system. Amounts of total RNA for RT-PCR pre-excluded the amplification of templates from contaminating genomic DNA at 35-PCR cycles. Staining of tissue sections with cresyl violet did not affect the performance of RT-PCR, and RNA-derived PCR products could be amplified using RNA extracted from tissue dissected with the LCM system.

\section{Discussion}

There are three major critical requirements for RNA analysis using PET: (a) Efficiency of RNA extraction
A
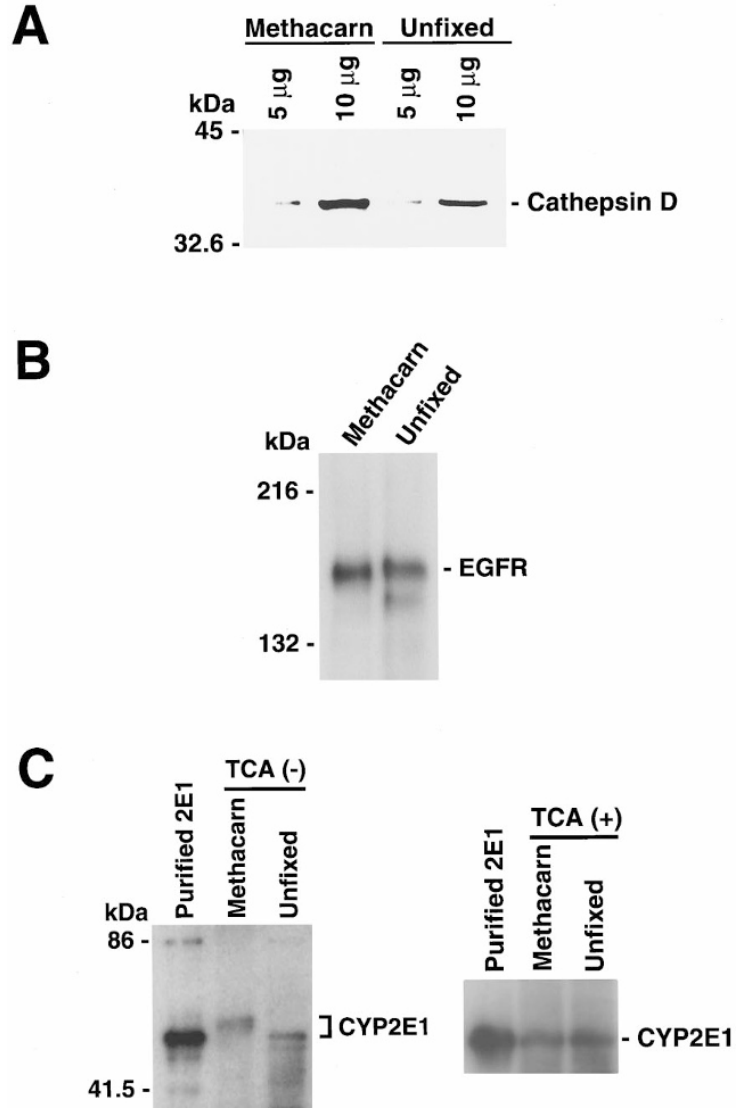

Figure 7.

Comparison of protein signals detected in methacarn-fixed PETs and unfixed frozen tissues by Western blotting. A, Cathepsin D-expression in 0.2-ppm ethinyl estradiol-treated mouse uterus. Either 5 or $10 \mu \mathrm{g}$ of protein lysate was subjected to $14 \%$ sodium dodecyl sulfate (SDS)-polyacrylamide-gel electrophoresis. B, EGFR expression in rat liver tissue. Each protein lysate $(10 \mu \mathrm{g})$ was subjected to $5 \%$ SDS-polyacrylamide gel electrophoresis. Relative band intensity of the protein derived from methacarn-fixed samples to unfixed frozen samples was $101.1 \pm 30.6 \%(n=4)$. C, CYP2E1-expression in rat liver tissue. Ten $\mu \mathrm{g}$ of protein lysate was subjected to $7.5 \%$ SDS-polyacrylamide-gel electrophoresis. Protein extracts were prepared either with or without TCAprecipitation of deparaffinized tissue section. Relative band intensity of the protein from methacarn-fixed samples to unfixed sample was $101.9 \pm 21.9 \%$ $(n=5)$. Purified 2E1: liver microsomal fraction isolated from the rat treated with acetone.

(Foss et al, 1994; Koopmans et al, 1993; Tyrrell et al, 1995); (b) Quality of extracted RNA (Finke et al, 1993; Rupp and Locker, 1988; Sato et al, 1991; Stanta and Schneider, 1991); (c) Noncontamination of genomic DNA (Ben-Ezra et al, 1991; Foss et al, 1994; Rupp and Locker, 1988; von Weizsäcker et al, 1991). In studies in which several fixation methods were examined for the feasibility of RT-PCR by judging the efficiency of amplification of RT products, non-cross-linking fixatives always gave superior results to formaldehydebased fixatives (Goldworthy et al, 1999; Koopmans et al, 1993; Tyrrell et al, 1995), although there are no differences in yields of extracted RNA (Foss et al, 1994). In the present study, however, the RNA yields from methacarn-fixed paraffin-embedded PC12 cells and rat liver tissue blocks were $56 \%$ and $46 \%$, respectively, of those from unfixed frozen counterparts, despite the fact that formaldehyde-based fixatives 


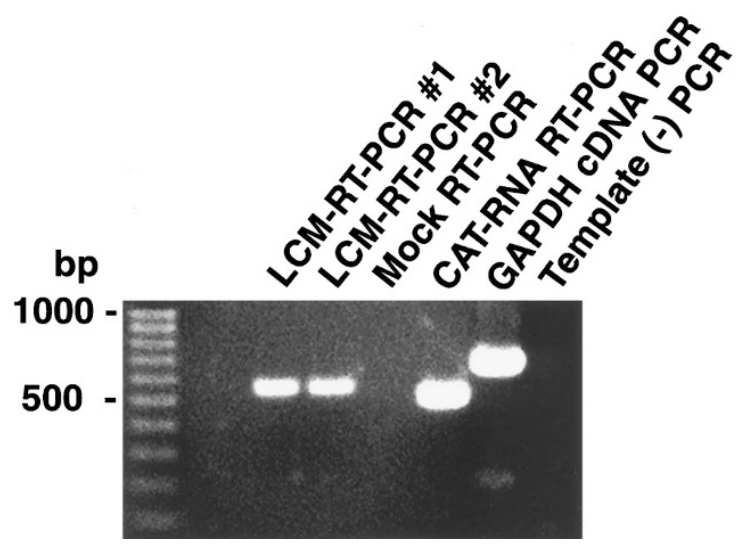

Figure 8

LCM-RT-PCR detection of HGFR RNA. Liver total RNA extracted from laser-microdissected tissue was used for RT-PCR. RT products were generated from 46 and $15 \mathrm{ng}$ of total RNA, respectively, for \#1 and \#2 PCRs.

gave poor RNA-yields by our simple extraction system. This result contrasts with the poor extraction yield of less than 1/10th of frozen tissue, obtained with PET after fixation in Carnoy's solution (Foss et al, 1994). In addition to the successful LCM-RT-PCR result with 15-50 ng total RNA, the RNA yields of around $50 \mathrm{ng}$ from a $1-\mathrm{mm}^{2}$ area obtained in our study underline the possibility of quantitative mRNA analysis using cDNA competitive RT-PCR, as described previously (Shibutani et al, 1998). Although acetone-fixed tissue in the present study provided a superior RNA yield than methacarn-fixed tissue, a relatively high level of genomic DNA contamination in the RNA preparation may diminish its advantage in extraction efficiency.

Although experimental conditions generally differ from those used in our present study, total RNA extracted from formalin-fixed PET is usually degraded considerably (Finke et al, 1993; Rupp and Locker, 1988; Stanta and Schneider, 1991). On the other hand, in addition to the extraction efficiency, the integrity of extracted total RNA from our methacarn-fixed PC12 cells was comparable to that from unfixed frozen cells. Our reasonable RT-PCR results to amplify 300-700 base pair fragments of any RNA species, even those expressing low copy numbers, may prove the quality of extracted RNA from methacarn-fixed PETs. While specific mechanisms of fixation for RNA preservation are still at the level of hypotheses, the proteinprecipitating effects of methacarn fixation may lead to inactivation of endogenous RNases and/or masking of the mRNAs from RNase action by precipitation of surrounding ribosomal proteins in the cell. Although the 1.9-kb fragment of EGFR mRNA could be amplified in our methacarn-fixed liver tissue, the relatively weak signal of such a long sequence may suggest the undesirable effects of the paraffin-embedding procedure for the retention of large RNA molecules to be amplified (Goldworthy et al, 1999). The question of how long mRNAs are retained intact in PET should now be addressed. In the present study, mRNA, as well as protein could be analyzed with PET which had been prepared five months previously.

Genomic DNA contamination in total RNA preparations from PET, in contrast to frozen tissues or cell samples, is a widely reported finding in studies evaluating RNA analysis by RT-PCR (Ben-Ezra et al, 1991; Foss et al, 1994; Rupp and Locker, 1988; von Weizsäcker et al, 1991). In the present study, however, the concentration of contaminating genomic DNA in the total RNA preparation from methacarn-fixed rat liver PET was quite low and even better than the level from unfixed frozen tissue, suggesting the suitability for RT-PCR analysis. When genomic DNA sequence information was not available for the gene of interest, we employed validation experiments for each gene. At first, the maximum amount of total RNA for the RT reaction was determined by performing a mock RT reaction followed by PCR with the maximum of 35 cycles. Then the optimum cycle number was determined within the range of exponential amplification of RT products. By this two-step validation, mRNAspecific template amplification could be detected even where genomic sequence information was lacking. However, housekeeping genes, such as GAPDH in the present study, usually share homologous sequences with genomic pseudogenes, and such sequences in contaminating genomic DNA in total RNA preparations would be amplified preferentially by RTPCR (Foss et al, 1994; Hanauer and Mandel, 1984). Therefore, such housekeeping genes cannot be recommended for RT-PCR analysis using fixed PET.

For protein analysis, non-cross-linking fixatives, such as acetone, ethanol or modified Carnoy's solution, give clear protein bands for cytokeratin molecules (Conti et al, 1988). In the present study, acetone gave the best protein yield among fixatives, and an EGFR signal equivalent to that in unfixed tissue could be detected in acetone-fixed tissue. However, about a $13.5 \%$ reduction of protein yield was observed in our methacarn-fixed PET as compared to that from unfixed frozen tissue. Lipid extraction by such alcoholbased protein precipitating fixatives may result in the "diffusion artifact" of membrane-bound proteins, causing their immunohistochemical detection to be difficult (Gusterson et al, 1984; Judd and Britten, 1982; Ponder and Wilkinson, 1981). The slight loss in the protein yield in our methacarn-fixed PET may suggest such an extraction effect during fixation and subsequent paraffin embedding and deparaffin steps. However, we did not see any artificial fluctuation in the expression levels of membrane-bound proteins, such as CYP2E1 and EGFR, as compared with those from unfixed frozen tissues, suggesting that effects of lipid extraction could be negligible in methacarn-fixed PETs used to perform quantitative protein analysis. Furthermore, the polypeptide band of all examined proteins, in which two glycoproteins (cathepsin D and EGFR) were included, migrated to the positions of correct molecular size (17-170 kDa) in our methacarnfixed PET. This result indicates that proteins of any molecular size could be preserved as a biochemically intact form in such PETs, although some unknown 
modification on certain populations of polypeptides affecting the mobility in SDS-polyacrylamide gel would appear without TCA precipitation of the tissue samples. This was observed in the CYP2E1 signal in the present study.

In conclusion, methacarn-fixed PET may be particularly suitable for analysis of molecular events at the mRNA level in specific cell populations, especially in conjunction with LCM. Considering its advantages for immunohistochemical detection of proteins (Mitchell et al, 1985; Puchtler et al, 1970), tissue-embedding after methacarn-fixation should be recommended as a valuable approach for routine application, possibly in combination with immuno-LCM, a recently developed method which allows targeted mRNA analysis of immunophenotypically-defined cell populations (Fend et al, 1999).

\section{Materials and Methods}

\section{Fixatives}

A methacarn solution consisting of $60 \%$ (vol/vol) absolute methanol, $30 \%$ chloroform, and $10 \%$ glacial acetic acid was freshly prepared before fixation. Other fixatives used for comparison of extraction efficiency of RNA and protein were as follows: acetone; $4 \%$ paraformaldehyde in $0.1 \mathrm{M}$ phosphate-buffered saline (pH 7.4); 10\% neutral-buffered formalin; Ufix, a commercial fixative consisting of formalin and methanol (Sakura Fine Technical Company, Ltd., Tokyo, Japan); and Bouin's solution. Ultrapure water was used for preparation of fixatives after treatment with diethyl pyrocarbonate.

\section{Tissue and Cell Preparation}

After exsanguination from the abdominal aorta under ether anesthesia, F344 rats of both sexes were killed for removal of liver, kidneys, lungs, and brain. To facilitate fixation, tissues were trimmed to make 5-mm-thick slices. For comparison of methacarn with other fixatives on the extraction efficiency of RNA and protein/wet tissue weight, 1-mm-thick rat-liver slices weighing 50-60 mg were prepared for fixation. Ovariectomized female CBA mice fed a diet containing 0.2-ppm ethinyl estradiol for 10 days were also killed under ether anesthesia, and the uteri were removed. The organs and tissues were fixed in methacarn or other fixatives for one hour at $4^{\circ} \mathrm{C}$, except for acetone fixation at $-80^{\circ} \mathrm{C}$. For embedding, tissue samples were dehydrated three times for one hour in fresh $100 \%$ ethanol at $4^{\circ} \mathrm{C}$, immersed in xylene once for one hour and then three times for 30 minutes at room temperature, and immersed in hot paraffin $\left(60^{\circ} \mathrm{C}\right)$ three times for one hour. Liver tissue samples for estimation of RNA-yield/unit area were trimmed into cubes with a $3.0-\mathrm{mm}^{2}$-section area.

A rat pheochromocytoma-derived cell line, PC12, was grown in flasks as described previously (Shibutani et al, 1998) and harvested. Washed cells were counted and divided into $5 \times 10^{5}$ aliquots per tube, fixed either with methacarn or paraformaldehyde/0.1 M phos- phate buffer for 1 hour, and then dehydrated, placed in xylene, and embedded in paraffin.

\section{RNA and Protein Extraction}

Thin sections from paraffin-embedded tissue, 4 to10 $\mu \mathrm{m}$ in thickness, were prepared and deparaffinized with xylene three times for 10 minutes and placed in $100 \%$ ethanol for washing three times for 10 minutes. After brief air drying, tissue sections were lysed with RNA STAT-60 (Tel-Test "B", Inc., Friendswood, Texas) and total cytoplasmic RNA was isolated with isopropanol precipitation in the presence of glycogen at 2 $\mu \mathrm{g} / \mathrm{ml}$ as a carrier. For comparison of extraction efficiency of total RNA and protein between fixatives, PET blocks were directly deparaffinized, disintegrated with Mikro-Dismembrator S (B. Braun Biotech International $\mathrm{GmbH}$, Melsungen, Germany) for subsequent extraction. Isolated RNA was labeled with a RiboGreen RNA quantitation kit (Molecular Probes, Eugene, Oregon), and concentrations were estimated with a fluorescence spectrophotometer (F4010, Hitachi Company, Ltd., Tokyo, Japan). To estimate the purity of RNA, concentrations of contaminating DNA in the final preparation were measured using Hoechst 33258 Molecular Probes (Molecular Probes, Eugene, Oregon). Total RNA was also isolated from methacarn-fixed paraffin-embedded PC12 cells to compare the RNA yield and integrity with those for unfixed frozen cells. The integrity of total RNA was confirmed by resolution of $18 \mathrm{~S}$ and $28 \mathrm{~S}$ ribosomal RNA in $0.8 \%$ formaldehyde agarose gels stained with ethidium bromide.

Protein from deparaffinized tissue was solubilized and sonicated for 10 minutes in $2 \times$ SDS gel-loading buffer. For the Western blotting analysis of CYP2E1 in the rat liver, protein precipitation with 10\% TCA was performed before solubilization in SDS buffer. Concentrations were estimated with a NanoOrange protein quantitation kit (Molecular Probes, Eugene, Oregon).

\section{RT-PCR}

Single-strand DNA was generated from 15 to $1000 \mathrm{ng}$ of total RNA with random hexamers and the Superscript Preamplification System (Life Technologies, Inc., Rockville, Maryland). Hot start PCR was performed with Platinum Taq DNA polymerase (Life Technologies, Inc., Rockville, Maryland) in a total $20-\mu$ l volume. The cycle parameters for the PCR of the target fragments, sized smaller than one $\mathrm{kb}$, were typically $94^{\circ} \mathrm{C}$ for 1 minute, $55^{\circ} \mathrm{C}$ for 1 minute, and $72^{\circ} \mathrm{C}$ for 1 minute. Table 3 lists target genes, primer sets for PCR, and the expected sizes of the mRNAderived PCR products. Among the genes examined in the present study, information on exon-intron boundaries was available for $\alpha_{2 \mathrm{u}}$-globulin, PRL, GH, TSH $\beta$, CYP1A1, CYP2B1, CYP2B2, and CYP4A1 genes, and primers for such genes were designed to overlap two or more exons to distinguish PCR products derived from genomic DNA and mRNA. Under the prescribed 


\begin{tabular}{lcccc}
\hline & & \multicolumn{2}{c}{ Combination of primers } & \\
\cline { 2 - 4 } Gene & Accession No. & Upstream* & Downstream & Target size (bp) \\
\hline$\alpha_{2 \text { 2 }}$-G, rat & M26835 & $231-250$ & $715-734$ & $504 \dagger$ \\
CYP1A1, rat & X00469 & $1331-1348$ & $1650-1669$ & $339 \dagger$ \\
CYP2B1, rat & J00719 & $759-776$ & $1118-1136$ & $378 \dagger \ddagger$ \\
CYP4A1, rat & X07259 & $511-530$ & $880-899$ & $389 \dagger$ \\
EGFR, rat & M37394 & $154-179$ & $818-843$ & 693 \\
& & & $2029-2048$ & 1895 \\
GAPDH, rat & X02231 & $379-404$ & $1035-1060$ & 682 \\
GH, rat & V01237 & $29-48$ & $581-600$ & $572 \dagger$ \\
HGFR, rat & U65007 & $187-206$ & $727-746$ & 560 \\
PRL, rat & V01249 & $86-104$ & $565-584$ & $499 \dagger$ \\
RPL7, rat & X15013 & $327-346$ & $779-798$ & $472 \dagger$ \\
TSH $\beta$, rat & D00578 & $3-21$ & $300-319$ & $317 \dagger$ \\
TSHR, rat & M34842 & $3101-3120$ & $3815-3833$ & $733 \dagger$ \\
\hline
\end{tabular}

* Nucleotide numbers are shown for each gene according to the mRNA sequence registered to the GenBank/EMBL Data Bank with the accession number. $\dagger$ Actual PCR product will add 16 bases to the target fragment because of tag sequences including restriction site at the $5^{\prime}$-ends of primers. ¥ The set of CYP2B1 primers also amplifies 402 bp fragment of CYP2B2 cDNA (Geng and Strobel, 1995).

$\alpha_{2 u}-\mathrm{G}, \alpha_{2 u}-$ Globulin; CYP1A1, Cytochrome P450 enzyme 1A1; CYP2B1, Cytochrome P450 enzyme 2B1; CYP4A1, Cytochrome P450 enzyme 4A1; EGFR, Epidermal growth factor receptor; GAPDH, Glyceraldehyde-3-phosphate-dehydrogenase; GH, Growth hormone; HGFR, Hepatocyte growth factor receptor; PRL, Prolactin; RPL7, Ribosomal protein L7; TSH $\beta$, Thyrotropin $\beta$ subunit; TSHR, TSH receptor.

PCR conditions, only the shorter mRNA-derived cDNA was successfully amplified at the expense of any potential genomic DNA contamination (Foss et al, 1994). A primer set for CYP2B1 was also designed to detect the CYP2B2 gene (Geng and Strobel, 1995). In the liver, amplification of the genes for the following was tested: $\alpha_{2 \mathrm{u}}$-globulin, HGFR, TSH $\beta$, TSHR, PRL, GH, GAPDH, ribosomal protein L7, CYP1A1, CYP2B1, CYP2B2 and CYP4A1. In addition to the liver tissue, brain, lung, and kidney tissues were tested for amplification of HGFR and $\alpha_{2 \mathrm{u}}$-globulin. The PCR conditions for each gene were estimated by optimizing the amount of total RNA for the RT reaction, followed by the cycle number for PCR. The amount of total RNA for $\mathrm{RT}$ reaction was determined by negativity for PCR amplification of the template derived from contaminating genomic DNA by reverse transcriptase (-) mock RT-PCR at 35 cycles. The optimum cycle number for each gene was determined within the range of the exponential phase of PCR amplification using RT products.

In vitro transcribed RNA of the chloramphenicol acetyltransferase gene and its gene-specific primers included in the Superscript Preamplification System (Life Technologies, Inc., Rockville, Maryland) were used as a positive control for RT-PCR, with a predicted PCR product size of 523 base pairs. Positive and negative PCR controls were prepared using GAPDH primers with or without the GAPDH cDNA template in each PCR experiment.

\section{Immunoblotting Analysis}

Protein samples were subjected to SDS-polyacrylamidegel electrophoresis and then transferred to a polyvinylidine difluoride membrane (Millipore, Bedford, Massachusetts). After blocking with $0.2 \%$ casein (Merck,
Whitehouse Station, New Jersey), blots were incubated either with anti-rat $\alpha_{2 \mathrm{u}}$-globulin (the kind gift of Dr. Masahiro Takeyoshi), anti-rat CYP2E1 (Daiichi Chemical Company, Ltd., Tokyo, Japan), anti-human EGFR (Medical and Biological Laboratories Company, Ltd., Nagoya, Japan) or anti-mouse cathepsin D (Medical and Biological Laboratories Company, Ltd., Nagoya, Japan). Bound antibodies were detected with sheep anti-mouse Ig or donkey anti-rabbit Ig antibodies conjugated with horseradish peroxidase (Amersham Pharmacia Biotech $A B$, Uppsala, Sweden) and analyzed either with the Supersignal Chemiluminescent Substrate (Pierce Technology Corporation, Inc., New York, New York) or the BIO-RAD HRP color reagent (Hercules, California). Relative protein levels of EGFR and CYP2E1 in the methacarn-fixed rat liver PETs were estimated by analyzing the band intensities obtained by Western blots using the United States National Institute of Health image program. Values were calculated as percentages of unfixed frozen liver tissues.

\section{Laser Capture Microdissection}

Thin sections were mounted on diethyl pyrocarbonatetreated slide glasses, deparaffinized, dehydrated, stained with cresyl violet, and again dehydrated. Stained or unstained sections were used for microdissection using a pixel laser capture microscope system with an infrared diode laser (LM100, Olympus, Tokyo, Japan). Tissue sections were overlaid with a thermoplastic membrane mounted on optically transparent caps, and cells were captured with focal melting of the membrane through laser activation. After visual control of the completeness of dissection, the captured tissue was immersed in RNA STAT-60 for subsequent total RNA extraction. 


\section{Acknowledgements}

We thank Dr. Masahiro Takeyoshi for providing antirat $\alpha_{2 \mathrm{u}}$-globulin antibody and its purified antigen from urine.

\section{References}

Alvares K, Subbarao V, Sambasiva Rao M, and Reddy JK (1996). Ciprofibrate represses $\alpha_{2 u}$-globulin expression in liver and inhibits d-limonene nephrotoxicity. Carcinogenesis 17: 311-316.

Banks PM (1979). Diagnostic applications of an immunoperoxidase method in hematopathology. J Histochem Cytochem 27:1192-1194.

Ben-Ezra J, Johnson DA, Rossi J, Cook N, and Wu A (1991). Effect of fixation on the amplification of nucleic acids from paraffin-embedded material by the polymerase chain reaction. J Histochem Cytochem 39:351-354.

Conti CJ, Larcher F, Chesner J, and Aldaz CM (1988). Polyacrylamide gel electrophoresis and immunoblotting of proteins extracted from paraffin-embedded tissue sections. J Histochem Cytochem 36:547-550.

Dries V, von Both I, Muller, M Gerken G, Schirmacher P, Odenthal M, Bartenschlager R, Drebber U, Meyer zum Buschenfeld KH, and Dienes HP (1999). Detection of hepatitis C virus in paraffin-embedded liver biopsies of patients negative for viral RNA in serum. Hepatology 29:223-229.

Faliks D and Meyuhas O (1982). Coordinated regulation of ribosomal protein $\mathrm{mRNA}$ level in regenerating rat liver. Study with the corresponding mouse cloned cDNAs. Nucleic Acids Res 10:789-801.

Fend F, Emmert Buck MR, Chuaqui R, Cole K, Lee J, Liotta LA, and Raffeld M (1999). Immuno-LCM: laser capture microdissection of immunostained frozen sections for mRNA analysis. Am J Pathol 154:61-66.

Finke J Fritzen R, Ternes P, Lange W, and Dölken G (1993). An improved strategy and a useful housekeeping gene for RNA analysis from formalin-fixed, paraffin-embedded tissues by PCR. BioTechniques 14:448-453.

Foss RD, Guha Thakurta N, Conran RM, and Gutman P (1994). Effects of fixative and fixation time on the extraction and polymerase chain reaction amplification of RNA from paraffin-embedded tissue. Comparison of two housekeeping gene mRNA controls. Diagn Mol Pathol 3:148-155.

Geng J and Strobel HW (1995). Identification of inducible mixed function oxidase system in rat glioma C6 cell line. J Neurochem 65:554-563.

Goldworthy SM, Stockton PS, Trempus CS, Foley JF, and Maronpot RR (1999). Effects of fixation on RNA extraction and amplification from laser capture microdissected tissue. Mol Carcinog 25:86-91.

Guerrero RB, Batts KP, Brandhagen DJ, Germer JJ, Perez RG, and Persing DH (1997). Effects of formalin fixation and prolonged block storage on detection of hepatitis $C$ virus RNA in liver tissue. Diagn Mol Pathol 6:277-281.

Guerrero RB, Batts KP, Germer JJ, Perez RG, Wiesner RH, and Persing DH (1998). Reverse transcriptase-polymerase chain reaction fails to detect peripheral-blood hepatitis C RNA in formalin-fixed liver tissue. Liver Transpl Surg 4:455-460.
Gusterson B, Cowley G, and Smith J (1984). Cellular localisation of human epidermal growth factor receptor. J Cell Biol Int Rep 8:649-658.

Hanauer A and Mandel JL (1984). The glyceraldehyde-3phosphate dehydrogenase gene family: structure of a human cDNA and of an X chromosome linked pseudogene; amazing complexity of the gene family in the mouse. EMBO J 3:2627-2633.

Hara A, Sakai N, Yamada H, Yoshimi N, Tanaka T, and Mori H (1993). Immunoblot analysis of the placental form of glutathione S-transferase in protein extracted from paraffinembedded human glioma tissue. J Cancer Res Clin Oncol 119:493-496.

Ikeda K, Monden T, Kanoh T, Tsujie M, Izawa H, Haba A, Ohnishi T, Sekimoto M, Tomita N, Shiozaki H, and Monden M (1998). Extraction and analysis of diagnostically useful proteins from formalin-fixed, paraffin-embedded tissue sections. $\mathrm{J}$ Histochem Cytochem 46:397-403.

Ikeda S, Takasu M, Matsuda T, Kakinuma A, and Horio F (1997). Ascorbic acid deficiency decreases the renal level of kidney fatty acid-binding protein by lowering the $\alpha_{2 \mathrm{u}}$-globulin gene expression in liver in scurvy-prone ODS. J Nutr 127: 2173-2178.

Judd MA and Britten KJM (1982). Tissue preparation for the demonstration of surface antigens by immunoperoxidase techniques. Histochem J 14:747-753.

Kocarek TA, Kraniak JM, and Reddy AB (1998). Regulation of rat hepatic cytochrome $\mathrm{P} 450$ expression by sterol biosynthesis inhibition: inhibitors of squalene synthase are potent inducers of CYP2B expression in primary cultured rat hepatocytes and rat liver. Mol Pharmacol 54:474-484.

Koopmans M, Monroe SS, Coffield LM, and Zaki SR (1993). Optimization of extraction and PCR amplification of RNA extracts from paraffin-embedded tissue in different fixatives. J Virol Methods 43:189-204.

Liu Y, Tolbert EM, Sun AM, and Dworkin LD (1996). Primary structure of rat HGF receptor and induced expression in mesangial cells. Am J Physiol 40:F679-F688.

Mitchell D, Ibrahim S, and Gusterson BA (1985). Improved immunohistochemical localization of tissue antigens using modified methacarn fixation. J Histochem Cytochem 33:491-495.

Miyauchi I, Moriyama M, Zhang DY, and Abe K (1998). Further study of hepatitis $C$ virus RNA detection in formalinfixed, paraffin-embedded liver tissues by ligation-dependent polymerase chain reaction. Pathol Int 48:428-432.

Moulton BC and Khan SA (1992). Progestin and estrogen control of cathepsin $\mathrm{D}$ expression and processing in rat uterine luminal epithelium and stroma-myometrium. Proc Soc Exp Biol Med 201:98-105.

Neuhaus OW (1986). Renal reabsorption of low molecular weight proteins in adult male rats: $\alpha_{2 \mathrm{u}}$-globulin. Proc Soc Exp Biol Med 182:531-539.

Neuhaus OW (1992). Renal reabsorptive capacity for $\alpha_{2 u^{-}}$ globulin in the adult male rat. Proc Soc Exp Biol Med 200:85-89.

Omiecinski CJ, Redlich CA, and Costa P (1990). Induction and developmental expression of cytochrome P450IA1 messenger RNA in rat and human tissues: detection by the polymerase chain reaction. Cancer Res 50:4315-4321. 
Orstavik TB, Brandtzaeg P, Nustad K, and Pierce JV (1981). Effects of different tissue processing methods on the immunohistochemical localization of kallikrein in the pancreas. J Histochem Cytochem 29:985-988.

Ponder BA and Wilkinson MM (1981). Inhibition of endogenous tissue alkaline phosphatase with the use of alkaline phosphatase conjugates in immunohistochemistry. J Histochem Cytochem 29:981-984.

Prough RA, Webb SJ, Wu HQ, Lapenson DP, and Waxman DJ (1994). Induction of microsomal and peroxisomal enzymes by dehydroepiandrosterone and its reduced metabolite in rats. Cancer Res 54:2878-2886.

Puchtler H, Waldrop FS, Meloan SN, Terry MS, and Conner HM (1970). Methacarn (Methanol-Carnoy) fixation. Practical and theoretical considerations. Histochemie 21:97-116.

Rochefort H, Cavailles V, Augereau P, Capony F, MaudeIonde T, Touitou I, and Garcia M (1989). Overexpression and hormonal regulation of pro-cathepsin $\mathrm{D}$ in mammary and endometrial cancer. J Steroid Biochem 34:177-182.

Rognum TO, Brandtzaeg $\mathrm{P}$, Ørjasaeter $\mathrm{H}$, and Fausa $\mathrm{O}$ (1980). Immunohistochemistry of epithelial cell markers in normal and pathological colon mucosa. Comparison of results based on routine formalin- and cold ethanol-fixation methods. Histochemistry 67:7-21.

Rupp GM and Locker J (1988). Purification and analysis of RNA from paraffin-embedded tissues. BioTechniques 6:5660.
Sato Y, Mukai K, Furuya S, Kameya T, and Hirohashi S (1992). The AMeX method: a multipurpose tissue-processing and paraffin-embedding method. Extraction of protein and application to immunoblotting. Am J Pathol 140:775-779.

Sato Y, Mukai K, Furuya S, and Shimosato Y (1991). AMeX method: a multipurpose tissue-processing and paraffinembedding method. III. Extraction and purification of RNA and application to slot-blot hybridization analysis. J Pathol 163:81-85.

Shibutani M, Lazarovici $P$, Johnson AC, Katagiri $Y$, and Guroff G (1998). Transcriptional down-regulation of epidermal growth factor receptors by nerve growth factor treatment of PC12 cells. J Biol Chem 273:6878-6884.

Stanta G and Schneider C (1991). RNA extracted from paraffin-embedded human tissues is amenable to analysis by PCR amplification. BioTechniques 11:304-308.

Tyrrell L, Elias J, and Longley J (1995). Detection of specific mRNAs in routinely processed dermatopathology specimens. Am J Dermatopathol 17:476-483.

von Weizsäcker F, Labeit S, Koch HK, Oehlert W, Gerok W, and Blum HE (1991). A simple and rapid method for the detection of RNA in formalin-fixed, paraffin-embedded tissues by PCR amplification. Biochem Biophys Res Commun 174:176-180. 